\title{
लिंगआधारीत बजेट आणि वास्तवीकता
}

Gender-based budgeting and realism

\section{Dr. Rakshit Madan Bagde,}

Assistant Professor, Late. Mansaramji Padole Arts College, Ganeshpur Bhandara

ORCID iD - 0000-0002-7507-0244

Web of Science ResearcherID - AAF-2760-2020

SSRN - Author ID: 4770534

Authenticus ID - R-00J-YM2

Vidwan-ID : 221858

rakshitbagde@gmail.com

आधूनिक युगात लिंगआधारीत बजेट समाजाच्या विविध वर्गातील वाढत्या चेतनेचा प्रभाव आहे. भारतीय समाज हा विविध जाती, धर्म आणि पंथात विभागलेला आहे. या सर्वामध्ये समानतेची अपेक्षा आणि त्याला पुर्ण करण्याची चेतना निर्माण झालेली दिसून येते. भारतीय अर्थव्यवस्थेचा विचार करता हल्ली लिंग आधारीत बजेट ही संकल्पना पूढे आलेली आहे. यातून राष्ट्र आणि राज्य यांचे प्रयत्न आहे की, सरकारी योजनेचा लाभ हा समाजातील स्त्री वर्गापर्यंत पोहचावा आणि लिंग आधारीत पुरूष आणि स्त्री यातील विषमतेची दरी दूर केली जावी.

लिंगआधारीत बजेट याचा अर्थ स्त्रीयांकरीता वेगळे बजेट निर्माण करणे नसून मूख्य बजेट मध्येच समायोजन करून सामाजीक आणि लैंगीक विषमता दूर करण्याचा प्रयत्न करणे होय. प्रायोगीक स्थरावर प्रथमतः ऑस्ट्रेलिया मध्ये सन 1980 मध्ये लिंगआधारीत बजेट प्रस्तूत करण्यात आले होते. त्यानंतर मात्र सन 1989 मध्ये ब्रिटन मध्ये आणि सध्या जगातील 70 देशात लिंगआधारीत बजेट चा उपयोग केल्या जात आहे.

सध्या भारतात बदलत्या काळाच्या अनुसंगाने कल्याणाची जागा सशक्तिकरणाने घेतलेली आहे आणि त्यामूळेच देशात 9 व्या पंचवार्षीक योजनेत $30 \%$ रक्कम लिंगआधारीत बजेटच्या सूपाने स्त्री वर्गाकरीता विविध योजनेच्या स्वसूपात वितरीत करण्यात आले होते. सन 2001 ला भारत सरकारने ‘महिला सशक्तिकरण वर्ष’ घोषीत कसून देशात महिला विकासाला प्राधान्य देण्यात आले. 
देशात 16 मार्च 2012 ला प्रणव मुखर्जी द्वारा लोकसभेत सन 2012-13 बजट प्रस्तूत केले. याचा आढावा घेतल्यास याला उत्साही बजेट म्हणता येणार नाही.

2012-13 बजेट च्या मूख्य तरतूदी-

1) सबला योजने करीता 7500 कोटीची तरतूद करण्यात आली.

2) महिला शेतकरी वर्गाकरीता 3915 कोटीची तरतूद करण्यात आली.

3) महिला स्व-सहायता समूहाकरीता 300 कोटीची मदत करणे.

4) बाल आणि महिला कूपोषणाकरीता सोया प्रोटीनच्या सीमा शूल्कात $10 \%$ कपात.

5) महिला उत्पन्न कर मर्यादा 1.80 वरून 1.90 लाख करण्यात आली.

देशात राष्ट्रीय महिला आयोगाने 20 वर्ष पूर्ण केलेली आहेत. या दृष्टीकोनातून विचार करता देशात सन 1991 मध्ये 945 वर असलेला बाल-लिंगानुपात खाली घसरून सन 2011 मध्ये 914 वर आलेला आहे. सध्या देशात 32 करोड महिला प्रजनन सबंधीत रोगाने ग्रस्त आहेत. एका अंदाजा नूसार 27 कोटी महिला या 'प्रीमेंस्टुअल सिंड्रोम’ आजाराने ग्रस्त आहेत. देशातील ग्रामीण महिलांचा विचार करता $40 \%$ महिलांना ल्युकोरीया, अल्सर आणि गर्भासयाचा कैंसर या रोगाने ग्रस्त आहेत. राष्ट्रीय पोषण मॉनिटरींग ब्यूरो नूसार देशात 13 ते 15 वर्षातील तरूणींना 1620 कैलरी चे अन्न मिळत आहे जेक्टा की त्यांना 2050 कैलरीची आवश्यकता आहे. आमच्या देशात प्रती एक लाख जिवंत मूलाच्या बाळंतपणात 450 मातांना आपला जीव गमवावा लागतो आहे. त्यामूळेच स्वास्थ सूविधांच्या बाबतीत भारतात महिलांचा विकास हा अजूनही अपूर्ण आणि त्याहिपेक्षा चिंताजनक झालेला आहे. 
राष्ट्रीय महिला आयोगाच्या एका अहवाला नूसार, देशातील 612 जिल्हयात केलेल्या एका अध्ययनानुसार हल्ली अर्ध्यापेक्षा जास्त जिल्हयात कमी वयाच्या मूली, तरूणी आणि महिलांची तस्करी केली जात आहे. सीबीआय च्या अहवालानुसार भारतात वेश्यालयात 13 लाख महिला काम करीत असून एकटया मुंबईतील कामाठीपूरम मध्ये 70 हजार महिलांचा समावेश आहे. तसेच देशात शोषण आणि बलात्काराचे प्रकरण दरवर्षी वाढतच चालेले आहेत.

देशात कृषी क्षेत्रात स्त्रियांची भूमीका स्विकारून डॉ. स्वामीनाथन यांनी राज्यसभेत 'महिला कृषी हक्कधारी विधेयक' 2011 प्रस्तूत करण्याचा प्रस्ताव मांडला होता. या प्रस्तावात महिला शेतकय्यांकरीता अनुकूल वातावरण निर्माण करणे, पानी, कर्ज आणि साधने मीळण्यास मदत आणि जमीनीचे पट्टे देने या प्रमूख बार्बींचा यात समावेश होता. सध्या देशात $11 \%$ महिलांकडेच जमीनीचे पट्टे असून यातील सर्वात जास्त सिमांत आणि लहान शेतकरी आहेत. डॉ. स्वामीनाथन यांच्या पाच सुत्री कार्यक्रमाकडे लक्ष दिल्यास यातून महिलांना काही प्रमाणात फायदा होण्याची शक्यता आहे. परंतू देशात या पाच सुत्री कार्यक्रमाची अंमलबजावणी अत्यंत हळूवारपणे होत असल्याचे दिसून येते.

देशात महिला भुरुण हंत्या हा चिंतेचा विषय बनत चालेला आहे. सन 1986-2006 या काळात देशात 1 कोटी स्त्री भुण हंत्या करण्यात आलेली होती. याचे मूख्य कारण गरीबी सांगण्यात येत असले तरी ग्रामीण भागाच्या तूलनेत शहरी भाग हा स्त्री भुण हंत्येच्या बाबतीत पुढारलेला आहे. देशात प्रत्येकवर्षी 15 लाख जन्मलेल्या मूलीपैकी, 1.5 लाख मूली आपल्या पहील्या जन्मदिवसा अगोदर तर $25 \%$ मूली या आपल्या 15 व्या जन्मदिवसा अगोदर मरण पावतात.

भारतात स्त्रीयांचा साक्षरता दर पुरूषांच्या तूलनेत कमी राहीलेला आहे.एका अंदाजानुसार देशात $56 \%$ महिला निरक्षर असून पुरूषांचे प्रमाण यात $24 \%$ आहे. येणाया पाच वर्षात यात सुधारणा होण्याची शक्यता असली तरी देशातील सामाजीक आणि आर्थिक विषमता यात अडथळे 
निर्माण करीत आहे. सन 2007- 08 च्या राष्ट्रीय नमूना सर्वेक्षणाच्या अहवाला नूसार देशात गेल्या 20 वर्षात वेतनधारक महिलांची संख्या फक्त $15 \%$ असलेली दिसून येते. यातून देशातीत उरलेल्या $85 \%$ महिलांच्या विकासाचा मार्ग अजूनही अवरूद्ध असल्याचे निदर्शनास येते .

खय्या अर्थाने महिलांना शसक्त करायचे असेल तर, आता महिलांना सामाजीक अधिकार, शिक्षण, न्याय, स्वास्थ, प्रतिष्ठा आणि वित्तीय सुदृढता प्रदाण करण्याची गरज निर्माण झालेली आहे. महिलांना एकत्रीत करून, त्यांचे समूह तयार करून त्यांना तांत्रीक, संगठनात्मक आणि वाणिज्यीक क्षमता आणि सामर्थ्यांत सुधारणा करण्याकरीता नविन कार्यक्रम तयार करणे गरजेचे झाले आहे.

संदर्भ ग्रंथ -

योजना (2012) - ‘आजादी के 65 वर्ष' 538 योजना भवन, संसद मार्ग, नयी दिल्ली. अगस्त योजना (2012) - 'बजेट 2012-13' 538 योजना भवन, संसद मार्ग, नयी दिल्ली. अप्रैल योजना (2012) - 'स्त्री सशक्तीकरण' 538 योजना भवन, संसद मार्ग, नयी दिल्ली. जून कुसूक्षेत्र (2011) - ‘ग्रामीण महिला सशक्तिकरण' 655 निर्माण भवन, ग्रामीण विकास मंत्रालय, नयी दिल्ली. सितंबर. 